\title{
Infecções fúngicas invasivas em pacientes com lúpus eritematoso sistêmico juvenil
}

Tese apresentada à Faculdade de Medicina da Universidade de São Paulo para obtenção do título de Doutor em Ciências

Programa de Pediatria

Orientador: Prof. Dr. Clovis Artur Almeida da Silva

São Paulo 
Dados Internacionais de Catalogação na Publicação (CIP)

Preparada pela Biblioteca da

Faculdade de Medicina da Universidade de São Paulo

Creprodução autorizada pelo autor

Silva, Marco Felipe Castro da

Infecções fúngicas invasivas em pacientes com lúpus eritematoso sistêmico juvenil / Marco Felipe Castro da Silva. -- São Paulo, 2015.

Tese(doutorado)--Faculdade de Medicina da Universidade de São Paulo. Programa de Pediatria.

Orientador: Clovis Artur Almeida da Silva.

Descritores: 1.Infecção 2.Micoses 3.Lúpus eritematoso sistêmico 4.Estudos de coortes 5.Ensaio clínico 6.Criança 7.Glicocorticoides 8.Candidíase

9.Aspergilose 10.Criptococose 11.Histoplasmose 12.Paracoccidioidomicose 13.Prednisona

USP/FM/DBD-208/15 


\section{DEDICATÓRIA}

Aos meus pais, Helito Pereira da Silva e Maria de Fátima Castro da Silva, os quais sempre apoiaram minhas escolhas e nunca mediram esforços para oferecer o que fosse necessário à minha formação pessoal e profissional. Nada seria possível sem o apoio deles. 


\section{AGRADECIMENTOS}

Ao Prof. Dr. Clovis Artur Almeida da Silva, baiano incansável e patriarca da minha Família Reumatológica Paulistana, a quem tenho grande estima e admiração. Obrigado pela amizade (inclusive pelas conversas discordantes que tanto geraram ensinamento e me fizeram crescer), assim como pelo estímulo constante aos estudos, orientação e realização deste projeto.

Ao Dr. Carlos Nobre Rabelo Júnior, meu "irmão reumático" mais velho e eterno mestre, responsável direto pela minha decisão de fazer Reumatologia Pediátrica. Obrigado pela amizade, paciência, apoio incondicional, confiança no meu trabalho e no meu potencial (até quando eu mesmo chego a ficar em dúvida), além da disponibilidade e prontidão diuturnas para me substituir nas inúmeras vezes que precisei ir a São Paulo com o intuito de concretizar esta tese.

À amiga e braço-direito Mariana Paes Leme Ferriani, que esteve por perto durante toda a realização deste projeto, dividindo os sucessos e as angústias, desde à criação do banco de dados até a redação final.

Aos chefes, assistentes e pós-graduandos de todos os 10 serviços de Reumatologia Pediátrica do Estado de São Paulo participantes do presente estudo: Unidade de Reumatologia Pediátrica do Instituto da Criança da Faculdade de Medicina da USP (Adriana Maluf Elias Sallum, Cristina Miuki Abe Jacob, Gabriela Blay, Gabriela Nunes Leal, Gabriella Erlacher Lube de Almeida, Heloisa Helena de Souza Marques, João Domingos Montoni da Silva, Joaquim Carlos Rodrigues, Juliana Caires de Oliveira Achili Ferreira, Kátia Kozu, Laila Pinto Coelho, Lúcia Maria Arruda Campos, Luciana dos Santos Henriques, Magda Carneiro-Sampaio, Maria Helena Vaisbich, Mariana Paes Leme Ferriani, Nádia Emi Aikawa, Roberta Cunha Gomes, Victor Leonardo Saraiva Marques, Werther Brunow de Carvalho); Unidade 
de Reumatologia Pediátrica da UNIFESP (Ana Paula Sakamoto, Anandreia Simões Lopes, Cláudio Arnaldo Len, Daniela Petry Piotto, Giampaolo Faquin, Gleice Clemente, Maria Odete Esteves Hilário, Maria Teresa Terreri, Melissa Fraga, Octávio Augusto Bedin Peracchi, Vanessa Bugni); Divisão de Reumatologia da FMUSP (Eloisa Bonfá, Glauce Leão Lima, Juliane Paupitz, Rosa Maria Pereira); UNESP (Priscila R. Aoki, Juliana de Oliveira Sato, Silvana Paula Cardin, Taciana Albuquerque Pedrosa Fernandes); Irmandade da Santa Casa de Misericórdia de São Paulo (Andressa Guariento, Eunice Okuda, Maria Carolina dos Santos, Natali Weniger Spelling Gormenzano, Silvana Brasília Sacchetti); Universidade Estadual de Campinas (Maraísa Centeville, Renata Barbosa, Roberto Marini, Simone Appenzeller); Faculdade de Medicina de Ribeirão Preto - USP (Francisco Hugo Gomes, Gecilmara Salviatto Pileggi, Paola Pontes Pinheiro, Virgínia Paes Leme Ferriani), Hospital Infantil Darcy Vargas (Cássia Maria Passarelli Barbosa, Jonatas Libório, Luciana Tudech Pedro Paulo), Hospital Municipal Infantil Menino Jesus (Simone Lotufo, Tânia Caroline Monteiro de Castro) e PUC Sorocaba (Valéria C. Ramos). Muito obrigado pela disponibilidade, inclusão dos pacientes e enorme empenho dedicados à força-tarefa que foi coletar os dados deste projeto. Ele certamente não seria tão grandioso sem a ajuda de vocês.

Ao Dr. Ulysses Doria Filho, pela disponibilidade, paciência e análise estatística.

Às assistentes Dra. Adriana Maluf, Dra. Adriana de Jesus, Dra. Ana Paola Lotito, Dra. Elizabeth Canova, Dra. Lúcia Campos e Dra. Nádia Aikawa, pelos ensinamentos diários e pela amizade. Todas, sem exceção, são médicas extremamente dedicadas e competentes, sem as quais aprender Reumatologia Pediátrica seria ainda mais difícil.

Ao meu tio Vicente Teixeira, amigo e companheiro inseparável, pela amizade, abrigo, apoio, orientação cultural e culinária, ensinamentos profissionais e pessoais, tudo de extrema importância para meu 
crescimento. Obrigado por ter ajudado tanto desde a minha chegada a São Paulo até meu retorno a Fortaleza. Minha eterna gratidão a você.

À minha tia Lúcia Teixeira, pela amizade e apoio, desde as minhas primeiras semanas em São Paulo.

Aos meus irmãos, Fabíola, David e Rodrigo, por estarem sempre presentes ao longo desta jornada.

À Amanda Carneiro Donato, pelo enorme apoio, tornando mais leves e felizes vários momentos árduos desta trajetória, sobretudo na reta final.

Aos amigos do coração Andréa Lima, Anna Gabriella Costa, Felippe Borges, Isabela Corrêa, Izabella Tamira Galdino, Marcella Coelho, Márcia Cerqueira, Matheus Rios, Paulo Cesar Mesquita, Raquel Maia, Renata Rolim, Tárcilla Passos, Tereza Raquel Bessa, que sempre estiveram presentes, de perto ou de longe, me apoiando e me dando forças para seguir.

Aos amigos de São Paulo lone, Lígia Martini, Ulysses Formiga e Rodrigo, pela amizade, apoio e companhia em jantares, teatros e óperas. Afinal, numa jornada tão longa, também deve haver espaço para diversão.

Aos amigos Camila França, Érica Gomes e Vanessa Guissa, pela amizade, cooperação e união nesta trilha tão difícil que é a Pós-Graduação.

Aos complementandos contemporâneos Priscila Freire, Guilherme Trudes, Victor Marques, Izabel Buscatti, Maria Fernanda Giacomin, Roberta Gomes, Gabriela Viola e Daniela Mencaroni, que fizeram da complementação em Reumatologia Pediátrica um período cheio de aprendizado e companheirismo.

Às minhas queridas residentes, Ana Raquel Xavier Feitosa e Míria Paula Vieira Cavalcante, pessoas iluminadas que, além de ajudar no que fosse possível durante minhas ausências devido ao Doutorado, foram de inestimável valor para meu crescimento pessoal e profissional. Minha eterna gratidão por me proporcionarem a possibilidade de aprender a ensinar a arte da Reumatologia Pediátrica. 
Ao SAME, especialmente nas pessoas da Elza, Lucimeire e Luides, pela disponibilidade de acesso aos prontuários médicos, essenciais para 0 estudo.

À amiga Mariza Kazue Umetsu Yoshikawa, pela amizade, apoio e especialmente pelas contribuições na pesquisa bibliográfica.

Ao amigo Nivaldo Rocha, pela amizade espontânea, conversas, cafés e todas as importantes contribuições ao longo da complementação em Reumatologia Pediátrica e na Pós-Graduação no Instituto da Criança. 
"O sucesso nasce do querer, da determinaçãoe persistência em se chegar a um objetivo. Mesmo não atingindo $\sigma$ alvo, quem busca e vence obstáculos, no mínimo fará coisas admiráveis" 
"Todas as vitórías ocultam uma abdicação"

Simone de Beauvoir 
Esta dissertação está de acordo com as seguintes normas, em vigor no momento desta publicação:

Referências: adaptado de International Committee of Medical Journals Editors (Vancouver)

Universidade de São Paulo. Faculdade de Medicina. Serviço de Biblioteca e Documentação. Guia de apresentação de dissertações, teses e monografias. Elaborado por Anneliese Carneiro da Cunha, Maria Julia de A. L. Freddi, Maria F. Crestana, Marinalva de Souza Aragão, Suely Campos Cardoso, Valéria Vilhena. $2^{\mathrm{a}}$ ed.

São Paulo: Serviço de Biblioteca e Documentação; 2005.

Abreviaturas dos títulos dos periódicos de acordo com List of Journals Indexed in Index Medicus. 


\section{SUMÁRIO}

Lista de Abreviaturas e Siglas

Lista de Tabelas

Resumo

Abstract

1 INTRODUÇÃO........................................................................ 1

2 OBJETIVO............................................................................. 4

3 MÉTODO..................................................................................... 6

3.1. Desenho do Estudo e População.......................................... 7

3.2. Infecções Fúngicas Invasivas............................................ 8

3.3. Dados Demográficos, Características Clínico-laboratoriais, Atividade da doença, Dano cumulativo e Tratamento......................... 9

3.4. Análise Estatística.......................................................... 11

4 RESULTADOS........................................................................... 12

4.1. Prevalência e Caracterização das IFI no LESJ.......................... 13

4.2. LESJ com IFI versus LESJ sem IFI................................... 15

4.3. Análise Multivariada......................................................... 19

4.4. Análise de Subgrupos................................................... 20

5 DISCUSSÃO................................................................................. 21

6 CONCLUSÕES 25

7 REFERÊNCIAS........................................................................... 27

\section{ANEXOS}




\section{LISTA DE ABREVIATURAS E SIGLAS}

\begin{tabular}{|c|c|}
\hline $\mathrm{aCL}=$ & Anticorpos anticardiolipina \\
\hline $\mathrm{ACR}=$ & American College of Rheumatology \\
\hline cSLE = & Childhood-onset systemic lupus erythematosus \\
\hline$E D A=$ & Endoscopia digestiva alta \\
\hline EORTC/MSG= & $\begin{array}{l}\text { European Organization for Research and Treatment of } \\
\text { Cancer/Invasive Fungal Infections Cooperative Group and } \\
\text { the National Institute of Allergy and Infectious Diseases } \\
\text { Mycoses Study Group }\end{array}$ \\
\hline $\mathrm{HIV}=$ & Vírus da imunodeficiência humana \\
\hline $\mathrm{IC}=$ & Intervalo de confiança \\
\hline $\mathrm{IFI}=$ & Infecções fúngicas invasivas \\
\hline $\mathrm{LBA}=$ & Lavado broncoalveolar \\
\hline LES $=$ & Lúpus eritematoso sistêmico \\
\hline $\mathrm{LESa}=$ & Lúpus eritematoso sistêmico de início na idade adulta \\
\hline LESJ= & Lúpus eritematoso sistêmico juvenil \\
\hline $\mathrm{OR}=$ & Odds ratio \\
\hline $\mathrm{PCR}=$ & Proteína C-reativa \\
\hline RIFLE = & $\begin{array}{l}\text { Risk, Injury, Failure, Loss of kidney function and End- } \\
\text { stage kidney disease }\end{array}$ \\
\hline $\mathrm{SAF}=$ & Síndrome do anticorpo antifosfolípide \\
\hline SLEDAI-2K= & $\begin{array}{l}\text { Systemic Lupus Erythematosus Disease Activity Index } \\
2000\end{array}$ \\
\hline SLICC-ACR/DI= & $\begin{array}{l}\text { Systemic Lupus International Collaborating Clinics/ACR } \\
\text { Damage Index }\end{array}$ \\
\hline $\mathrm{TC}=$ & Tomografia computadorizada \\
\hline$H S=$ & Velocidade de hemossedimentação \\
\hline
\end{tabular}




\section{LISTA DE TABELAS}

Tabela 1- Características das infecções fúngicas invasivas (IFI) em 33/852 pacientes com lúpus eritematoso sistêmico juvenil (LESJ)

Tabela 2- Dados demográficos e manifestações clínicas atuais em 852 pacientes com lúpus eritematoso sistêmico juvenil (LESJ) agrupados de acordo com a presença de infecções fúngicas invasivas (IFI).

Tabela 3- Escores de atividade/dano cumulativo da doença e exames laboratoriais atuais em 852 pacientes com lúpus eritematoso sistêmico juvenil (LESJ) agrupados de acordo com a presença de infecções fúngicas invasivas (IFI)

Tabela 4- Terapia medicamentosa atual em 852 pacientes com lúpus eritematoso sistêmico juvenil (LESJ) agrupados de acordo com a presença de infecções fúngicas invasivas (IFI)

Tabela 5- Análise de regressão logística para avaliar fatores de risco para infecções fúngicas invasivas (IFI) em 852 pacientes com lúpus eritematoso sistêmico juvenil (LESJ) 


\section{RESUMO}

Silva MF. Infecções fúngicas invasivas em pacientes com lúpus eritematoso sistêmico juvenil [tese]. São Paulo: Faculdade de Medicina, Universidade de São Paulo; 2015.

Introdução: As infecções são importantes causas de morbidade e mortalidade em pacientes com lúpus eritematoso sistêmico juvenil (LESJ). No entanto, estudos avaliando somente infecções fúngicas invasivas (IFI) em pacientes com LESJ são restritos a relatos de casos ou série de casos, sem qualquer avaliação sistemática dos possíveis fatores de risco ou desfechos associados. A escassez de dados referentes às IFI em pacientes com LESJ e seu impacto sobre as características da doença em uma grande população levou ao desenvolvimento deste estudo multicêntrico. Objetivos: Estudar a prevalência, fatores de risco e mortalidade de IFI em pacientes com LESJ. Método: Um estudo de coorte multicêntrico retrospectivo foi realizado com 852 pacientes com LESJ de 10 Serviços de Reumatologia Pediátrica do Estado de São Paulo. Uma reunião foi realizada e todos os pesquisadores foram treinados para o preenchimento do banco de dados. As IFI foram diagnosticadas de acordo com as definições revisadas pelo grupo de consenso EORTC/MSG (comprovadas, prováveis ou possíveis). Foram coletados dados acerca de dados demográficos, características clínico-laboratoriais, atividade da doença (SLEDAI-2K), dano cumulativo (SLICC/ACR-DI) e tratamento, além de características e complicações das IFI. Resultados: IFI foram diagnosticadas em 33/852 (3,9\%) pacientes com LESJ. IFI comprovadas foram diagnosticadas em 22 pacientes, IFI prováveis em 5 e IFI possíveis em 6. Os tipos de IFI encontradas foram: candidíase em 20 pacientes, aspergilose em 9, criptococose em 2, histoplasmose disseminada em um e paracoccidioidomicose em um. A mediana de duração da doença foi menor ( 1,0 vs. 4,7 anos, $p<0,0001)$, com maiores escores de SLEDAl-2K atual [19,5 (0-44) vs. $2(0-45), p<0,0001]$ e dose atual de prednisona [50 (10-60) vs. 10 (2-90) mg/dia, $p<0,0001]$ em pacientes com IFI em comparação com os pacientes sem IFI. A frequência de óbito foi maior no grupo com IFI ( $51 \%$ vs. $6 \%, p<0,0001)$. A análise de regressão logística revelou que SLEDAI-2K atual $(O R=1,108, I C 95 \%=1,057-$ $1,163, p<0,0001)$, dose atual de prednisona (OR=1,046, IC 95\%=1,021-1,071; $p<0,0001)$ e duração da doença $(\mathrm{OR}=0,984$, IC $95 \%=0,969-0,998, \mathrm{p}=0,030)$ foram fatores de risco independentes para IFI ( $R^{2}$ Nagelkerke 0,425$)$. Conclusão: Este foi o primeiro estudo que caracterizou IFI em pacientes com LESJ. Identificou-se que a atividade da doença e uso de glicocorticoides foram os principais fatores de risco para estas infecções potencialmente graves, principalmente nos primeiros anos de curso da doença e com uma elevada taxa mortalidade.

Descritores: Infecção; Micoses; Lúpus eritematoso sistêmico; Estudos de coortes; Ensaio clínico; Criança; Glicocorticoides; Candidíase; Aspergilose; Criptococose; Histoplasmose; Paracoccidioidomicose; Prednisona. 


\section{ABSTRACT}

Silva MF. Invasive fungal infections in juvenile systemic lupus erythematosus patients [thesis]. São Paulo: “Faculdade de Medicina, Universidade de São Paulo"; 2015.

Introduction: Infections are an important cause of morbidity and mortality in childhoodonset systemic lupus erythematosus (CSLE) patients. However, studies evaluating solely invasive fungal infections (IFI) in cSLE patients are restricted to case reports or case series without any systematic evaluation of the possible associated risk factors and outcome in pediatric lupus population. The scarcity of data regarding IFI in cSLE patients and its impact on disease characteristics in a large population led to the development of this multicenter study. Objective: To study the prevalence, risk factors and mortality of IFI in cSLE patients. Methods: A retrospective multicenter cohort study was performed in 852 cSLE patients from 10 Pediatric Rheumatology services. An investigator meeting was held and all participants received database training. IFI were diagnosed according to EORTC/MSG Consensus Group criteria (proven, probable and possible). Demographic data, clinical, laboratorial, disease activity (SLEDAI-2K), cumulative damage (SLICC/ACR-DI) and treatment were collected. IFI were characterized and its outcome were also evaluated. Results: IFI were observed in 33/852 (3.9\%) cSLE patients. Proven IFI was diagnosed in 22 cSLE patients, probable IFI in 5 and possible IFI in 6. Types of IFI were: 20 candidiasis, 9 aspergillosis, 2 cryptococcosis, one disseminated histoplasmosis and one paracoccidioidomycosis. The median of disease duration was lower ( 1.0 vs. 4.7 years, $p<0.0001)$, with a higher current SLEDAI-2K [19.5 (0-44) vs. $2(0-45), p<0.0001]$ and current prednisone dose [50 (10-60) vs. $10(2-90) \mathrm{mg} /$ day, $\mathrm{p}<0.0001]$ in patients with IFI compared to those without IFI. The frequency of death was higher in the former group (51\% vs. $6 \%, p<0.0001)$. Logistic regression analysis revealed that current SLEDAI-2K (OR=1.108; $95 \% \mathrm{Cl}=1.057-1.163$; $\mathrm{p}<0.0001)$, prednisone current dose $(\mathrm{OR}=1.046 ; 95 \% \mathrm{Cl}=1.021-1.071 ; \mathrm{p}<0.0001)$ and disease duration $(\mathrm{OR}=0.984 ; 95 \% \mathrm{Cl}=0.969-0.998 ; \mathrm{p}=0.03)$ were independent risk factors for IFI $\left(R^{2}\right.$ Nagelkerke 0.425). Conclusion: This was the first study that characterized IFI in cSLE patients. We identified that disease activity and glucocorticoid use were the main risk factors for these life-threatening infections, mainly in the first years of disease course and with a high rate of fatal outcome.

Descriptors: Infection; Mycoses; Systemic lupus erythematosus; Cohort studies; Clinical trial; Child; Glucocorticoids; Candidiasis; Aspergillosis; Cryptococcosis; Histoplasmosis; Paracoccidioidomycosis; Prednisone. 
O lúpus eritematoso sistêmico (LES) juvenil (LESJ) é uma doença sistêmica, crônica, de etiologia multifatorial envolvendo herança multigênica e fatores ambientais ${ }^{1}$, caracterizada por produção de anticorpos auto reativos e alterações do sistema imunológico ${ }^{2,3,4}$. É uma doença rara, com incidência anual nos Estados Unidos estimada em 0,4 a 0,6 casos/100.000 crianças e adolescentes ${ }^{5}$, sendo excepcional antes dos cinco anos de idade ${ }^{6}$. Estima-se que 15 a $20 \%$ dos casos de LES têm início durante a infância ou adolescência ${ }^{7}$. Há predileção pelo sexo feminino e a proporção varia de 4:3 em pacientes com idade de início da doença durante a primeira década de vida a 9:1 com início durante segunda década de vida ${ }^{8}$.

O LES é o protótipo de doença autoimune sistêmica de herança poligênica, caracterizada por perda da tolerância imunológica a vários autoantígenos e formação de imunocomplexos que se depositam nos tecidos com conseqüente inflamação em diferentes órgãos e sistemas, conforme evidenciado em estudos realizados no nosso serviço ${ }^{9,10,11,12,13}$. O distúrbio imunológico do lúpus envolve apoptose dos linfócitos $B$ e linfócitos $T$ do sistema imune adaptativo, elementos do sistema imune inato como células dendríticas, deficiências de proteínas do sistema complemento e ainda polimorfismos das interleucinas, dos receptores Fc das imunoglobulinas e alterações no processo de apoptose celular ${ }^{2,4,14,15}$.

Infecções são importantes causas de morbidade e mortalidade em pacientes com LESJ ${ }^{16,17,18,19,20,21,22,23,24,25,26}$. Os fatores de risco mais importantes associados à infecção grave na população com lúpus estão relacionados tanto à doença em si (duração da doença, atividade da doença, linfopenia, leucopenia e hipocomplementemia) quanto ao seu tratamento (glicocorticoides e drogas imunossupressoras) ${ }^{16,18,19,22}$.

As infecções são causadas por vírus e bactérias ${ }^{16,18,19,25,26}$ na maioria dos casos, e com menor frequência por agentes oportunistas, tais como fungos $^{22}$. Infecções fúngicas são causadas principalmente por Candida spp., Pneumocystis jirovecii e Cryptococcus neoformans no LES de início na idade adulta (LESa) ${ }^{27}$. No entanto, estudos avaliando unicamente infecções fúngicas invasivas (IFI) em pacientes LESJ, como candidíase, aspergilose, 
criptococose e histoplasmose são restritas a relatos de casos e séries de $\operatorname{casos}^{16,17,18,20,21,22,23,24,26}$, sem qualquer avaliação sistemática dos possíveis fatores de risco associados.

A escassez de dados acerca das IFI em pacientes com LESJ, bem como seu impacto em características da doença e dos pacientes em uma representativa população de lúpus, estimulou a realização deste estudo multicêntrico. 
1. Avaliar a prevalência das IFI em pacientes com LESJ.

2. Avaliar a possível associação entre a presença de IFI em pacientes com LESJ com: dados demográficos, características clínicolaboratoriais, atividade e dano cumulativo da doença e tratamento. 


\subsection{Desenho do Estudo e População}

Este é um estudo de coorte multicêntrico retrospectivo, que incluiu todos os 1.017 pacientes com LESJ acompanhados em 10 serviços terciários de referência em Reumatologia Pediátrica do Estado de São Paulo, Brasil. Cento e sessenta e cinco pacientes não foram incluídos por: prontuários incompletos ou não encontrados $(n=96)$, doença do tecido conjuntivo indiferenciada com 3 ou menos critérios do American College of Rheumatology $(A C R)^{28}(n=43)$, lúpus eritematoso cutâneo isolado $(n=11)$, lúpus eritematoso neonatal $(n=8)$, lúpus induzido por drogas $(n=5)$ e outras doenças auto-imunes $(n=2)$. Nenhum paciente teve neoplasia hematológica, transplante de medula óssea ou infecção por vírus da imunodeficiência humana (HIV). Os 852 pacientes restantes constituíram o grupo de estudo.

Todos os pacientes preencheram os critérios do $\mathrm{ACR}^{28}$, com início da doença antes dos 18 anos de idade ${ }^{29}$ e idade atual até 25 anos. IFI foram diagnosticadas de acordo com a definição revisada pelo European Organization for Research and Treatment of Cancer/Invasive Fungal Infections Cooperative Group and the National Institute of Allergy and Infectious Diseases Mycoses Study Group (EORTC/MSG) Consensus Group $^{21,30,31}$, e candidíase oral e vulvovaginal foram excluídas. O Comitê de Ética em Pesquisa local de cada instituição aprovou o estudo.

Uma reunião foi realizada com os pesquisadores envolvidos neste estudo em 29 setembro de 2012 na cidade de São Paulo para apresentar o banco de dados, decidir definições consensuais e discutir outros parâmetros importantes a serem estudados. Os dados foram coletados entre novembro de 2012 e outubro de 2014. Os prontuários médicos dos pacientes foram cuidadosamente revistos de acordo com um extenso protocolo padronizado, incluindo dados demográficos, características clínicas, exames laboratoriais e dados terapêuticos, além de características e complicações das IFI. 


\subsection{Infecções Fúngicas Invasivas}

As recentes definições de IFI foram utilizadas e classificam o diagnóstico em três diferentes níveis de probabilidade em pacientes imunocomprometidos: comprovado, provável e possível. IFI comprovadas requerem o isolamento do fungo em tecido ou alguma situação especial descrita, como o isolamento do fungo numa amostra estéril. IFI prováveis requerem a presença de um fator de risco do hospedeiro (por exemplo, neutropenia e/ou uso de agentes imunossupressores), um critério clínico (síndrome clínica ou alterações laboratoriais sugestivas, como por exemplo febre prolongada, hepatoesplenomegalia, adenomegalia, pneumopatia, sinusopatia, meningoencefalite, sepse, osteomielite, tomografia computadorizada mostrando lesões focais em sistema nervoso central ou ressonância nuclear magnética evidenciando realce meníngeo) além de evidência micológica através de teste direto (cultura, citologia ou microscopia) ou teste indireto (detecção do antígeno ou componente da parede celular). IFI possíveis são definidas como a presença de fator de risco do hospedeiro associado a alterações clinico-laboratoriais sugestivas na ausência de evidência micológica ${ }^{21,30,31}$. Infecção por Pneumocystis jirovecci não foi incluída nessa definição.

As IFI descritas nos pacientes com LESJ foram: candidíase, aspergilose, histoplasmose, criptococose, paracoccidioidomicose, coccidioidomicose e mucormicose. Tipo e sítio de infecção das IFI, assim como isolamento do fungo, complicações relacionadas e terapia antifúngica, foram também avaliados. 


\subsection{Dados Demográficos, Características Clínico-laboratoriais, Atividade da doença, Dano cumulativo e Tratamento}

Os dados demográficos incluíram gênero, raça, idade atual, idade de início do LESJ e duração da doença. Manifestações clínicas do LES foram definidas como: sintomas constitucionais (febre e perda de peso), envolvimento do sistema retículo-endotelial (adenomegalia, hepatomegalia e esplenomegalia), lesões mucocutâneas (eritema malar ou discoide, fotossensibilidade, úlcera oral ou nasal, vasculite e alopécia), envolvimento músculo-esquelético (artrite não erosiva e miosite), serosite (pleurite e pericardite), nefrite (proteinúria $\geq 0,5 \mathrm{~g} / 24 \mathrm{~h}$, presença de cilindros celulares, hematúria persistente $\geq 5$ hemácias por campo e leucocitúria persistente $\geq 5$ leucócitos por campo), anormalidades hematológicas [anemia hemolítica auto-imune, leucopenia (contagem de leucócitos $<4.000 / \mathrm{mm}^{3}$ ), linfopenia (linfócitos $<1.500 / \mathrm{mm}^{3}$ ) e trombocitopenia (contagem de plaquetas $<100.000 / \mathrm{mm}^{3}$ ) em duas ou mais ocasiões, na ausência de drogas ou infecção]. Lúpus neuropsiquiátrico incluiu 19 síndromes de acordo com os critérios de classificação do $\mathrm{ACR}^{32}$. Síndrome do anticorpo antifosfolípide (SAF) foi diagnosticada de acordo com a presença de trombose arterial ou venosa associada à positividade para anticorpos antifosfolípides ${ }^{33}$.

Hipertensão arterial foi definida como pressão sistólica ou diastólica maior ou igual ao percentil 95 para gênero, idade e peso em 3 ou mais ocasiões $^{34}$. Lesão renal aguda foi determinada pelo aumento súbito na creatinina sérica acima de $2 \mathrm{mg} / \mathrm{dl}^{35}$ ou segundo os critérios modificado do Risk, Injury, Failure, Loss of kidney function and End-stage kidney disease $(\mathrm{RIFLE})^{36}$. A doença renal crônica foi definida como anormalidades estruturais ou funcionais do rim com duração $\geq 3$ meses (com ou sem diminuição da taxa de filtração glomerular) ou taxa de filtração glomerular $<60 \mathrm{ml} / \mathrm{min} / 1,73 \mathrm{~m}^{2}$ com duração $\geq 3$ meses $^{37}$. Terapia substitutiva renal (hemodiálise, diálise peritoneal, hemofiltração e/ou transplante renal) também foi avaliada. 
A avaliação laboratorial incluiu velocidade de hemossedimentação (VHS), proteína C-reativa (PCR), hemograma completo, ureia e creatinina séricas, urina I e proteinúria de 24 horas. Níveis de complemento (C3, C4 e $\mathrm{CH} 50$ ), anticorpo anti-DNA nativo e anticorpos anticardiolipina (aCL) $\operatorname{lgM}$ e IgG foram realizados em cada centro e os valores de corte foram considerados válidos. Anticoagulante lúpico foi detectado de acordo com as diretrizes da Sociedade Internacional de Trombose e Hemostasia ${ }^{38}$.

Atividade da doença e dano cumulativo foram mensurados através do Systemic Lupus Erythematosus Disease Activity Index 2000 (SLEDAI-2K) $^{39}$, que pode variar de 0 a 105, e do Systemic Lupus International Collaborating Clinics/ACR Damage Index (SLICC-ACR/DI) ${ }^{40}$, respectivamente. Este é um escore validado no LESJ e foi realizado nos pacientes com duração da doença acima de seis meses, podendo variar de 0 a 47. $O$ dano cumulativo corresponde à mudança irreversível, relacionada a atividade persistente da doença, medicamentos utilizados ou comorbidades. Episódios repetidos foram pontuados duas vezes somente se a mesma lesão ocorresse com intervalo mínimo de seis meses (por exemplo, dois episódios de acidente vascular cerebral, com intervalos acima de seis meses entre estes receberam pontuação 2 do SLICC/ACR-DI).

Também foram obtidos dados sobre o tratamento atual (prednisona, pulsoterapia com metilprednisolona, difosfato de cloroquina, sulfato de hidroxicloroquina, metotrexato, azatioprina, ciclosporina, micofenolato mofetil, ciclofosfamida endovenosa, gamaglobulina endovenosa, rituximab e plasmaférese). As doses cumulativas de prednisona, metilprednisolona intravenosa, glicocorticoide total e ciclofosfamida endovenosa também foram calculadas.

Os pacientes foram divididos em dois grupos para a avaliação das manifestações clínicas atuais, assim como parâmetros laboratoriais e tratamento: pacientes que tiveram IFI (avaliados durante o diagnóstico da infecção) e pacientes sem IFI (avaliados na última consulta). 


\subsection{Análise Estatística}

Os resultados foram apresentados como número (\%) para variáveis categóricas e mediana (variação) ou média \pm desvio padrão para variáveis contínuas. As variáveis categóricas foram comparadas através do teste do qui-quadrado de Pearson ou teste exato de Fisher. As variáveis contínuas foram comparadas pelo teste $t$ ou teste de Mann-Whitney. Os níveis de significância da variável independente foram fixados em 5\% ( $p<0,05)$. Correção de Holm-Bonferroni para comparações múltiplas foi realizada, ajustando o nível de significância para $p<0.0015$. Análise de regressão logística (Backward Stepwise) foi realizada para identificar fatores de risco para IFI. 


\subsection{Prevalência e Caracterização das IFI no LESJ}

IFI foram observadas em 33/852 (3,9\%) pacientes com LESJ, dos quais 22 tiveram IFI comprovadas, $5 \mathrm{IFI}$ prováveis e $6 \mathrm{IFI}$ possíveis. Em 6 pacientes com LESJ a doença fúngica invasiva ocorreu no início da doença, nos quais a mediana do SLEDAI-2K atual foi de 28 (24-41). Os tipos de IFI encontradas foram: candidíase em 20 pacientes, aspergilose em 9, criptococose em 2 (uma meningite criptocócica e uma criptococose pulmonar), histoplasmose disseminada em um e paracoccidioidomicose em um. Nenhum paciente apresentou coccidioidomicose ou mucormicose (Tabela 1). Pelo menos um sítio de infecção foi evidenciado em cada paciente: pulmão em 13, trato urinário em 8 , esôfago em 8 , sangue em 4 , sistema nervoso central em 3 e disseminado em 3 . Vinte e um foram hospitalizados em unidade de terapia intensiva pediátrica. As complicações mais freqüentes das IFI foram sepse em 18 e insuficiência renal aguda em 16 pacientes. Dos 17 óbitos, 15 tinham sepse e 14 apresentavam lesão renal aguda. Terapia substitutiva renal foi requerida em 3 pacientes, todos eles com aspergilose invasiva. A mediana da sobrevida desde o diagnóstico das IFI até o óbito foi de 30 (15-95) dias. As IFI mais comuns nos pacientes que faleceram foram aspergilose em 8 e candidíase em 7 pacientes.

Trinta e dois pacientes receberam pelo menos uma terapia antifúngica: 26 fluconazol, 10 anfotericina $\mathrm{B}, 4$ itraconazol, 3 voriconazol, um cetoconazol e um micafungina. Apenas um paciente não recebeu tratamento antifúngico devido ao diagnóstico post mortem. Vinte e um pacientes (63\%) foram submetidos a antibioticoterapia antes das IFI, notadamente $10(30 \%)$ pacientes com candidíase. A mediana da dose de prednisona atual nos pacientes que faleceram foi significantementemente maior quando comparados aos sobreviventes [60 (25-60 vs. 40 (10-60) mg/dia, p=0,014]. 
Tabela 1 - Características das infecções fúngicas invasivas (IFI) em 33/852 pacientes com lúpus eritematoso sistêmico juvenil (LESJ)

\begin{tabular}{|c|c|c|}
\hline IFI em pacientes com LESJ, $n=33$ & \multicolumn{2}{|c|}{ Critério micológico } \\
\hline IFI comprovada, $\mathrm{n}=22(67)$ & \multicolumn{2}{|c|}{ Isolamento do fungo } \\
\hline \multirow[t]{6}{*}{ Candidíase, $n=13$ (39) } & Urina $9(\mathrm{n}=6)$ & $\begin{array}{l}\text { Candida albicans }(n=4) \\
\text { Candida spp. }(n=1) \\
\text { Non-albicans yeast }(n=1)\end{array}$ \\
\hline & Sangue $\mathbb{\Upsilon}(\mathrm{n}=2)$ & $\begin{array}{l}\text { Candida albicans }(\mathrm{n}=1) \\
\text { Candida tropicalis }(\mathrm{n}=1)\end{array}$ \\
\hline & Biópsia de esôfago ( $n=2)$ & Candida spp. $(\mathrm{n}=2)$ \\
\hline & Esôfago $\ddagger(n=1)$ & Candida spp. $(\mathrm{n}=1)$ \\
\hline & 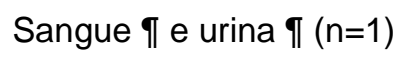 & Candida albicans $(\mathrm{n}=1)$ \\
\hline & $\begin{array}{l}\text { Urina \ e LBA \ }(n=1) \\
\text { Pulmão } \ddagger(n=3)\end{array}$ & $\begin{array}{l}\text { Candida albicans }(\mathrm{n}=1) \\
\text { Aspergillus spp. }(\mathrm{n}=3)\end{array}$ \\
\hline \multirow{2}{*}{ Aspergilose, $n=6$ (18) } & $\begin{array}{l}\text { Pulmão e cerebelo } ¥ \\
(n=1)\end{array}$ & Aspergillus spp. $(\mathrm{n}=1)$ \\
\hline & Miocárdio $\ddagger(n=1)$ & Aspergillus spp. $(\mathrm{n}=1)$ \\
\hline Criptococose, $n=2(6)$ & 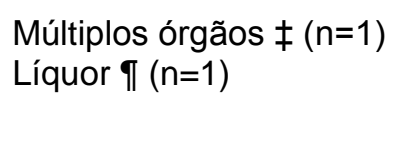 & $\begin{array}{l}\text { Aspergillus spp. }(\mathrm{n}=1) \\
\text { Cryptococcocus } \\
\text { neoformans }(\mathrm{n}=1)\end{array}$ \\
\hline Histoplasmose, $\mathrm{n=1}$ (3) & $\begin{array}{l}\text { Pulmão }(n=1) \\
\text { Linfonodo abdominal e } \\
\text { jejuno } ¥(n=1)\end{array}$ & $\begin{array}{l}\text { Cryptococcous spp. }(\mathrm{n}=1) \\
\text { Histoplasma capsulatum } \\
(\mathrm{n}=1)\end{array}$ \\
\hline Candidíase, $\mathrm{n}=2(6)$ & LBA $(n=2)$ & Candida albicans $(\mathrm{n}=2)$ \\
\hline Aspergilose, $n=2(6)$ & $\begin{array}{l}\text { Exame micológico } \\
\text { indireto }(n=1)\end{array}$ & $\begin{array}{l}\text { Galactomanana sérica } \\
\text { aumentada }(n=1)\end{array}$ \\
\hline & $\operatorname{LBA}(n=1)$ & Aspergillus fumigatus $(\mathrm{n}=1)$ \\
\hline Paracoccidioidomicose, $n=1(3)$ & $\begin{array}{l}\text { Exame micológico } \\
\text { indireto }(n=1)\end{array}$ & $\begin{array}{l}\text { Contraimunoeletroforese } \\
(\mathrm{n}=1)\end{array}$ \\
\hline IFI possível, n=6 (18) & \multirow{3}{*}{\multicolumn{2}{|c|}{$\begin{array}{l}\text { Alteração clínica ou laboratorial na ausência de } \\
\text { evidência micológica } \\
\text { Esofagite por Candida na } \operatorname{EDA}(n=5) \\
\text { Nódulo com halo de atenuação em vidro fosco em } \\
\text { pulmão direito }(T C) \text { resolvido após anfotericina B e } \\
\text { itraconazol }(n=1)\end{array}$}} \\
\hline Candidíase, n=5 (15) & & \\
\hline Aspergillose, $n=1$ (3) & & \\
\hline
\end{tabular}

I: Cultura, ‡: Isolamento na necrópsia, LBA: Lavado broncoalveolar, EDA: Endoscopia digestiva alta, TC: Tomografia computadorizada 


\subsection{LESJ com IFI versus LESJ sem IFI}

Dados demográficos e manifestações clínicas atuais estão na Tabela 2, e atividade da doença, escore de dano cumulativo e exames laboratoriais atuais em 852 pacientes com LESJ de acordo com a presença de IFI estão apresentados na Tabela 3.

Após a correção de Holm-Bonferroni para comparações múltiplas, as medianas do tempo de duração da doença ( 1,0 vs. 4,7 anos, $p<0,0001)$ e da idade atual $(14,5$ vs. 17,0 anos, $p<0,0001)$ foram significantemente menores nos pacientes com LESJ com IFI comparadas com os pacientes que não apresentaram IFI. As freqüências de sintomas constitucionais ou envolvimento do sistema retículo-endotelial foram significantemente maiores em pacientes que tiveram IFI em comparação com aqueles sem IFI ( $76 \%$ vs. $8 \%, p<0,0001$ ), assim como: envolvimento mucocutâneo (67\% vs. $13 \%, p$ $<0,0001$ ), comprometimento músculo-esquelético ( $36 \%$ vs. $0 \%, p<0,0001$ ), serosite $(39 \%$ vs. $0 \%, p<0,0001)$, manifestações neuropsiquiátricas $(36 \%$ vs. $8 \%, p<0,0001)$, nefrite ( $66 \%$ vs. $19 \%, p<0,0001)$ e hipertensão arterial (58\% vs. $12 \%, p<0,0001$ ) (Tabela 2 ). As medianas de SLEDAI-2K no início da doença [24 (2-45) vs. 14 (0-58), $p<0,0001]$ e de SLEDAI-2K atual [19,5 (0-44) vs. 2 (0-45), p <0,0001], assim como a frequência de complicações como insuficiência renal aguda ( $48 \%$ vs. $3 \%, p<0,0001)$ e óbito $(51 \%$ vs. $6 \%, p<0,0001$ ) foram mais altas em pacientes com LESJ e IFI (Tabela 3 ). 
Tabela 2 - Dados demográficos e manifestações clínicas atuais em 852 pacientes com lúpus eritematoso sistêmico juvenil (LESJ) agrupados de acordo com a presença de infecções fúngicas invasivas (IFI)

\begin{tabular}{|c|c|c|c|}
\hline Variáveis & $\begin{array}{c}\text { Com IFI } \\
(n=33)\end{array}$ & $\begin{array}{l}\text { Sem IFI } \\
(\mathrm{n}=819)\end{array}$ & $\boldsymbol{P}$ \\
\hline \multicolumn{4}{|l|}{ Dados demográficos } \\
\hline Gênero feminino & $29(88)$ & $703(86)$ & 1,000 \\
\hline Caucasiano, $n=830$ & $19 / 33(58)$ & $573 / 797(72)$ & 0,075 \\
\hline Idade ao diagnóstico de LESJ, anos, $\mathrm{n}=846$ & $12,4(5,1-17,2)$ & $11,8(0,2-17,8)$ & 0,159 \\
\hline Duração da doença, anos, $n=846$ & $1,0(0-7,3)$ & $4,7(0-23,4)$ & $<0,0001^{*}$ \\
\hline Idade atual, anos, $n=849$ & $14,5(6-20)$ & $17,0(2-25,9)$ & $<0,0001^{*}$ \\
\hline \multicolumn{4}{|l|}{ Manifestações clínicas atuais } \\
\hline $\begin{array}{l}\text { Sintomas constitucionais/envolvimento } \\
\text { do sistema reticuloendotelial, } n=850\end{array}$ & 25/33 (76) & $68 / 817(8)$ & $<0,0001^{*}$ \\
\hline Febre, $\mathrm{n}=849$ & 24/33 (73) & $35 / 816(4)$ & $<0,0001^{*}$ \\
\hline Perda ponderal $>2 \mathrm{~kg}, \mathrm{n}=824$ & $8 / 33(24)$ & $25 / 791(3)$ & $<0,0001^{*}$ \\
\hline Linfadenopatia, $n=848$ & 6/32 (19) & $8 / 816(1)$ & $<0,0001^{*}$ \\
\hline Hepatomegalia, $n=850$ & $9 / 33(27)$ & $15 / 817(2)$ & $<0,0001^{*}$ \\
\hline Esplenomegalia, $n=850$ & $3 / 33(9)$ & $5 / 817(1)$ & 0.003 \\
\hline Envolvimento mucocutâneo, n=850 & $22 / 33(67)$ & 108/817(13) & $<0,0001^{*}$ \\
\hline Envolvimento Musculoesquelético, $n=850$ & $12 / 33(36)$ & $0 / 817(0)$ & $<0,0001^{*}$ \\
\hline Serosites $n=848$ & $13 / 33(39)$ & $0 / 815(0)$ & $<0,0001^{*}$ \\
\hline Envolvimento neuropsiquiátrico, $\mathrm{n}=847$ & $12 / 33(36)$ & $65 / 814(8)$ & $<0,0001^{*}$ \\
\hline Nefrite, $n=831$ & $21 / 32(66)$ & 152/799 (19) & $<0,0001^{*}$ \\
\hline Trombose auto-imune atual (SAF), $\mathrm{n}=835$ & \multicolumn{2}{|c|}{ Complicações } & 0,012 \\
\hline Hipertensão arterial, $\mathrm{n}=843$ & 19/33 (58) & $100 / 810(\mathbf{1 2})$ & $<0,0001^{*}$ \\
\hline Lesão renal aguda, $n=841$ & $16 / 33(48)$ & $21 / 808(3)$ & $<0,0001^{*}$ \\
\hline Insuficiência renal crônica, n=842 & $3 / 33(9)$ & $24 / 809$ (3) & 0,084 \\
\hline Óbito, $n=845$ & $17 / 33(\mathbf{5 1})$ & $50 / 812(6)$ & $<0,0001^{*}$ \\
\hline
\end{tabular}


Tabela 3 - Escores de atividade/dano cumulativo da doença e exames laboratoriais atuais em 852 pacientes com lúpus eritematoso sistêmico juvenil (LESJ) agrupados de acordo com a presença de infecções fúngicas invasivas (IFI)

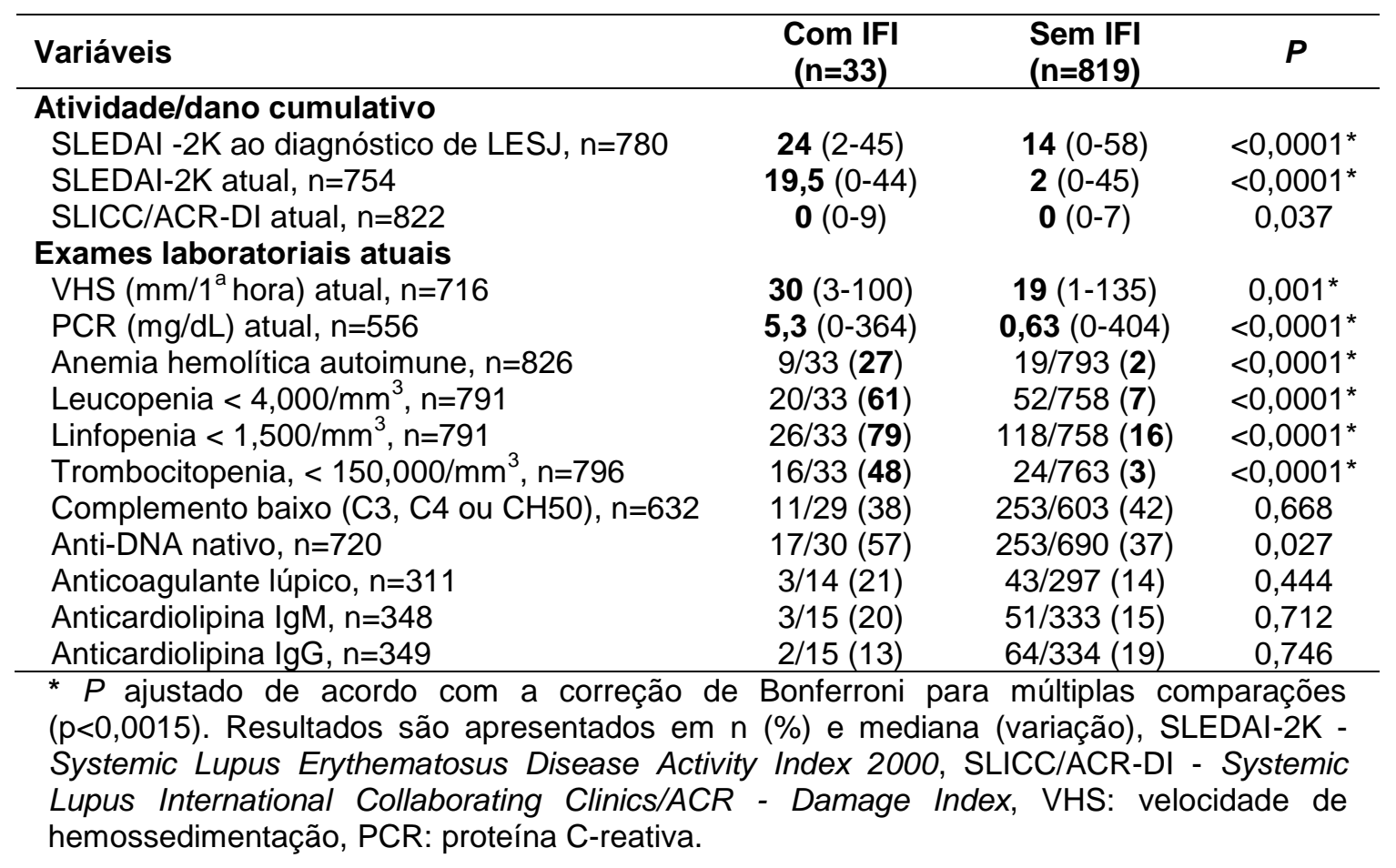

A terapia medicamentosa atual dos 852 pacientes com LESJ de acordo com a presença de IFI é ilustrada na Tabela 4. Após a correção de Holm-Bonferroni para comparações múltiplas, as freqüências dos seguintes tratamentos foram significantemente maiores em pacientes com IFI quando comparados aos sem IFI: pulsoterapia com metilprednisolona (61\% vs. $7 \%$, p <0,0001), ciclofosfamida endovenosa (27\% vs. $5 \%$, p <0,0001), imunoglobulina endovenosa ( $39 \%$ vs. $1 \%, p<0,0001)$ e plasmaferese $(12 \%$ vs. $0 \%, p<0,0001)$. Imunoglobulina endovenosa foi administrada para pacientes com infecções fúngicas graves.

A mediana da dose de prednisona atual também foi significantemente maior nos pacientes com LESJ com IFI em comparação com aqueles sem IFI [50 (10-60) vs. $10(2-90) \mathrm{mg} / \mathrm{dia}, p<0,0001]$. As doses cumulativas de 
prednisona $[15,77 \quad(0,24-35,6)$ vs. $19,08 \quad(0,12-105,51) \quad \mathrm{g}, \mathrm{p}=0.089]$,

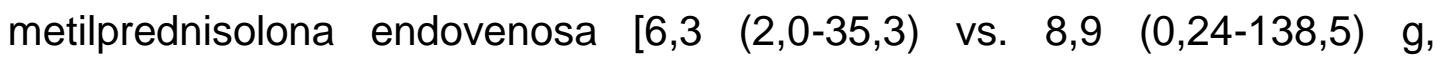
$\mathrm{p}=0,354]$ e de glicocorticoide total $[20,9(3,6-71,7$ vs. $26,07(0,42-205,54) \mathrm{g}$, $p=0,285]$ foram similares entre os pacientes com LESJ que tiveram e que não tiveram IFI. Após correção de Holf-Bonferroni para múltiplas comparações, a mediana da dose cumulativa de ciclofosfamida também não atingiu significância estatística em ambos os grupos $[2,8(0,6-12,8)$ vs. 7,0 $(0,26-93,0) \mathrm{g}, \mathrm{p}=0,002]$.

Tabela 4 - Terapia medicamentosa atual em 852 pacientes com lúpus eritematoso sistêmico juvenil (LESJ) agrupados de acordo com a presença de infecções fúngicas invasivas (IFI)

\begin{tabular}{|c|c|c|c|}
\hline Variáveis & $\begin{array}{c}\text { Com IFI } \\
(n=33)\end{array}$ & $\begin{array}{l}\text { Sem IFI } \\
(n=819)\end{array}$ & ${ }^{*} \boldsymbol{P}$ \\
\hline Anti-inflamatório não-hormonal, $n=843$ & $3 / 33(9)$ & $43 / 810(5)$ & 0,418 \\
\hline \multicolumn{4}{|l|}{ Glicocorticoides } \\
\hline Prednisona, $n=844$ & 29/33 (88) & 639/811 (79) & 0,208 \\
\hline Dose atual, $\mathrm{mg} / \mathrm{dia}, \mathrm{n}=669$ & $50(10-60)$ & $10(2-90)$ & $<0,0001^{*}$ \\
\hline Dose atual, $\mathrm{mg} / \mathrm{kg} / \mathrm{dia}, \mathrm{n}=648$ & $1,0(0,1-2,0)$ & $0,2(0,02-4,7)$ & $<0,0001^{*}$ \\
\hline Dose cumulativa, $\mathrm{g}, \mathrm{n}=710$ & $15,77(0,24-35,6)$ & $19,08(0,12-105,51)$ & 0,089 \\
\hline Metilprednisolona endovenosa, $n=841$ & $20 / 33(61)$ & $61 / 808(7)$ & $<0,0001^{*}$ \\
\hline Dose cumulativa, $g, n=470$ & $6,3(2,0-35,3)$ & $8,9(0,24-138,5)$ & 0,354 \\
\hline Glicocorticoide total cumulativo, $g, n=688$ & $20,9(3,6-71,7)$ & $26,7(0,42-205,54)$ & 0,285 \\
\hline Antimaláricos, $\mathrm{n}=841$ & $21 / 33(64)$ & $548 / 808(68)$ & 0,614 \\
\hline Difosfato de cloroquina, $n=816$ & $8 / 33(24)$ & $123 / 783(16)$ & 0,191 \\
\hline Sulfato de hidroxicloroquina, $n=818$ & $13 / 33(39)$ & $440 / 785(56)$ & 0,059 \\
\hline Imunosupressores, $\mathrm{n}=845$ & 23/33 (70) & $499 / 812(61)$ & 0,339 \\
\hline Azatioprina, $n=842$ & 14/33 (42) & 284/809 (35) & 0,389 \\
\hline Ciclosporina, $n=845$ & $4 / 33(12)$ & $25 / 812(3)$ & 0,023 \\
\hline Metotrexato, $n=844$ & 3/33 (9) & $70 / 811(9)$ & 0,759 \\
\hline Micofenolato mofetil, $n=845$ & $7 / 33(21)$ & $111 / 812(14)$ & 0,206 \\
\hline Ciclofosfamida endovenosa, $\mathrm{n}=844$ & $9 / 33(27)$ & $42 / 811(5)$ & $<0,0001^{*}$ \\
\hline Dose cumulativa, $g, n=306$ & $2,8(0,6-12,8)$ & $7,0(0,26-93,0)$ & 0,002 \\
\hline \multicolumn{4}{|l|}{ Outros } \\
\hline Imunoglobulina endovenosa, $n=843$ & 13/33 (39) & $7 / 810(1)$ & $<0,0001^{*}$ \\
\hline Rituximabe, $n=843$ & 1/33 (3) & $1 / 810(0)$ & 0,077 \\
\hline Plasmaferese, $n=845$ & 4/33 (12) & $3 / 812(0)$ & $<0,0001^{*}$ \\
\hline
\end{tabular}




\subsection{Análise Multivariada}

A análise de regressão logística revelou que SLEDAI-2K atual (OR=1,108, IC $95 \%=1,057-1,163, p<0,0001$ ), dose de prednisona atual (OR=1,046, IC $95 \%=1,021-1,071 ; \quad p<0,0001)$ e duração da doença (OR=0,984, IC $95 \%=0,969-0,998, \quad \mathrm{p}=0,030$ ) foram fatores de risco independentes para IFI. O R² de Nagelkerke foi 0,425. (Tabela 5).

Tabela 5 - Análise de regressão logística para avaliar fatores de risco para infecções fúngicas invasivas (IFI) em 852 pacientes com lúpus eritematoso sistêmico juvenil (LESJ)

\begin{tabular}{lcccc}
\hline Variável independente & $\mathrm{OR}$ & $\mathrm{IC} \mathrm{95 \%}$ & $\mathrm{R}^{2}$ & $\mathrm{P}$ \\
\hline SLEDAI-2K atual & 1,108 & $1,057-1,163$ & 0,425 & $<0,0001^{*}$ \\
$\begin{array}{l}\text { Prednisona, dose atual, } \\
\text { mg/dia }\end{array}$ & 1,046 & $1,021-1,071$ & - & $<0,0001^{*}$ \\
$\begin{array}{l}\text { Duração da doença } \\
\text { *P<0,05. SLEDAl-2K - Systemic Lupus Erythematosus Disease }\end{array}$ & 0,984 & $0,969-0,998$ & - & $0,030^{*}$ \\
$\begin{array}{l}\text { Odds ratio, IC - Intervalo de confiança, } \mathrm{R}^{2}-\mathrm{R}^{2} \text { de Nagelkerke } \\
\text { Odex }\end{array}$ & &
\end{tabular}

Duas outras análises de regressão logística foram realizadas, excluindo-se os pacientes que utilizaram ciclosporina e ciclofosfamida. As mesmas três variáveis independentes (SLEDAI-2K atual, dose de prednisona atual e duração da doença) foram fatores de risco para desenvolvimento de IFI nos dois modelos avaliados:

1. A análise de regressão logística excluindo os casos que usaram ciclosporina mostrou que SLEDAI-2K atual $(\mathrm{OR}=1,126$, IC $95 \%=1,071$ $1,183, p<0,0001)$, dose de prednisona atual $(O R=1,038$, IC $95 \%=1,014$ $1,064 ; p=0,002)$ e duração da doença $(O R=0,983$, IC 95\%=0,967-0,999, $\mathrm{p}=0,036$ ) também foram fatores de risco independentes para IFI. O $R^{2}$ de Nagelkerke foi 0,416.

2. A análise de regressão logística excluindo os casos que usaram ciclofosfamida mostrou que SLEDAI-2K atual $(O R=1,112$, IC $95 \%=1,056$ $1,171, p<0,0001)$, dose de prednisona atual $(O R=1,035$, IC $95 \%=1,009$ - 
$1,060 ; p=0,007)$ e duração da doença (OR=0,981, IC 95\%=0,965-0,996, $p=0,016)$ foram fatores de risco independentes para $I F I . O R^{2}$ de Nagelkerke foi 0,343 .

\subsection{Análise de Subgrupos}

Análise posterior de um subgrupo de 33 pacientes com IFI e 66 pacientes sem IFI (selecionados de forma randômica dos 819 sem IFI) que apresentaram duração da doença similares $[1(0-7,33)$ vs. $1(0-7,33)$ anos, $\mathrm{p}=0,772]$ mostrou maiores medianas de SLEDAI-2K atual [19,5 (0-44) vs. 4 $(0-45), p<0,0001]$ e de dose atual da prednisona [50 (10-60) vs. $20(5-80)$ $\mathrm{mg} / \mathrm{dia}, \quad p<0.0001]$ no primeiro grupo. A frequência de óbito foi significantemente maior nos pacientes com LESJ com IFI em comparação com aqueles sem essa infecção ( $51,5 \%$ vs. $20 \%, p=0,001)$. 
Este estudo evidenciou que as doenças fúngicas invasivas em pacientes com LESJ foram infecções potencialmente fatais e que ocorreram predominantemente no início da doença.

A vantagem do presente estudo foi a inclusão de uma grande população de LESJ acompanhada em 10 serviços terciários do Estado de São Paulo, utilizando um banco de dados padronizado para minimizar viéses. As definições de IFI foram baseadas em opinião de especialistas e um consenso internacional ${ }^{30,31}$. Esses critérios foram utilizados em ensaios clínicos e estudos epidemiológicos ${ }^{41}$. Além disso, candidíase localizada (como a oral e vulvovaginal) ${ }^{30}$ foi excluída, uma vez que não é invasiva e geralmente tratada rapidamente.

Entretanto, uma das limitações do presente estudo foi o desenho retrospectivo, com possibilidade de alguns dados incompletos nos prontuários. Os níveis de imunoglobulina não foram avaliados, impossibilitando uma interpretação definitiva a respeito de imunodeficiências primárias associadas ao lúpus pediátrico ${ }^{3,42}$. Além disso, apesar do uso da correção de Holm-Bonferroni para comparações múltiplas há colinearidade das variáveis utilizadas para diferenciar entre os parâmetros das IFI e da doença.

Infecções são uma importante causa de morbidade e mortalidade em pacientes com LESJ e foram evidenciadas entre $37 \%$ e $57 \%{ }^{18,19,22}$. Doenças fúngicas invasivas ocorrem mais freqüentemente em pacientes imunodeprimidos com câncer hematológico, transplante de medula óssea ${ }^{31}$ e infecção pelo $\mathrm{HIV}^{43}$. A literatura referente a IFI em adultos com LES consiste de relatos de casos e séries de casos, com prevalência variando entre 0,64 e 4,8\% ${ }^{43,44,45,46,47,48}$, semelhante à presente população estudada com lúpus de início na faixa etária pediátrica.

Nesta casuística, a maioria das IFI ocorreram nos primeiros anos da doença. Uma recente revisão sistemática sobre doença fúngica invasiva, utilizando os mesmos critérios para categorizar estas infecções ${ }^{30} \mathrm{em}$ pacientes com LESa, mostrou que as IFI foram diagnosticadas com uma mediana de dois anos de duração da doença ${ }^{43}$. Cerca de um quinto das 
crianças e adolescentes com LESJ tiveram doenças fúngicas invasivas no início da doença, uma freqüência maior do que a observada nos pacientes adultos com LES de início recente ${ }^{43}$. Todos eles tiveram atividade grave da doença (SLEDAI-2K>20), reforçando a observação anterior de que a infecção pode exacerbar, mimetizar ou induzir recidivas da doença ${ }^{18,21}$. Assim como nesta população estudada, altos escores de atividade da doença também foram relatados no início das IFI em pacientes adultos com LES $^{41,44,45,46}$.

Trato respiratório inferior foi o principal local de IFI em crianças e adolescentes, em contraste com adultos, nos quais há um predomínio de acometimento do sistema nervoso central ${ }^{43}$. Com relação às síndromes clínicas, a maior parte dos casos pediátricos foi composta por candidíase e aspergilose, contrastanto com os adultos, nos quais a criptococose parece ser a doença fúngica invasiva mais prevalente ${ }^{44,48}$. Candidíase sistêmica foi observada em 2,35\% dos nossos pacientes com LESJ, uma frequência comparável à reportada na população geral em quatro hospitais terciários da cidade de São Paulo, Brasil (4\%) ${ }^{49}$.

Alta taxa de mortalidade foi provavelmente mais relacionada à sepse e à lesão renal aguda, complicações muito prevalentes nos pacientes deste estudo $^{43}$. A própria atividade da doença pode cursar com linfopenia que pode ser agravada pela terapia citotóxica. Além disso, a administração de antibioticoterapia prévia às infecções fúngicas ocorreram em dois terços dos nossos pacientes, particularmente nos que tiveram candidíase, o que é similar à frequência relatada em pacientes com LESa ${ }^{50}$.

Pacientes com LESJ usualmente requerem maiores doses de glicocorticoide quando comparados aos pacientes com LESa ${ }^{51}$. De fato, altas doses atuais de glicocorticoide foram identificadas como um dos mais importantes fatores de risco para IFI em pacientes com LESJ, sem nenhuma associação com as doses cumulativas de glicocorticoide, e independentemente do uso da utilização de ciclosporina ou ciclofosfamida. Apesar disso, o uso concomitante de agentes imunossupressores nestes 
pacientes têm sido associado à diminuição da imunidade celular, com conseqüente linfopenia e risco de infecção grave ${ }^{41}$.

Terapia antifúngica foi iniciada prontamente em todos LESJ, com excecção de um paciente, principalmente fluconazol para candidíase e anfotericina B ou voriconazol para outras $\mathrm{IFI}^{43}$.

Apesar do tratamento antifúngico, metade dos pacientes com LESJ morreram, sugerindo que devam ser estabelecidas novas diretrizes para profilaxia com agentes antifúngicos na população com LESJ. Estes aspectos têm sido priorizados na prática clínica dos pacientes com câncer pediátrico em uso de imunossupressores ${ }^{52,53}$.

Além disto, estudos prospectivos e multicêntricos (como coorte de incepção) para avaliação de métodos diagnósticos sensíveis e específicos para o reconhecimento precoce da IFI, assim como desenvolvimento de protocolos de tratamento em pacientes com LESJ serão necessários. 
1. IFI foram raramente evidenciadas em pacientes com LESJ.

2. Atividade atual da doença e uso atual de glicocorticoide foram os principais fatores de risco para estas infecções graves, ocorrendo principalmente no início da doença e com uma elevada taxa de mortalidade. 


\section{REFERÊNCIAS}


1. Vidotto JP, Pereira LA, Braga AL, Silva CA, Sallum AM, Campos LM, Martins LC, Farhat SC. Atmospheric pollution: influence on hospital admissions in paediatric rheumatic diseases. Lupus 2012; 21: 526-33.

2. Carneiro-Sampaio M, Liphaus BL, Jesus AA, Silva CA, Oliveira JB, Kiss $\mathrm{MH}$. Understanding systemic lupus erythematosus physiopathology in the light of primary immunodeficiencies. J Clin Immunol 2008; 28(Suppl 1): 34-41.

3. Jesus AA, Liphaus BL, Silva CA, Bando SY, Andrade LE, Coutinho A, Carneiro-Sampaio M. Complement and antibody primary immunodeficiency in juvenile systemic lupus erythematosus patients. Lupus 2011; 20:1275-84.

4. Marks SD, Tullus K. Autoantibodies in systemic lupus erythematosus. Pediatr Nephrol 2012; 27: 1855-68.

5. Denardo BA, Tucker LB, Miller LC, Szer IS, Schaller JG. Demography of a regional pediatric rheumatology patient population. Affiliated Children's Arthritis Centers of New England J Rheumatol 1994; 21: 1553-61.

6. Gottlieb BS, llowite NT. Sistemic lupus erythematosus in children and adolescents. Pediatrics in review 2006; 22: 323-30.

7. Klein-Gitelman M, Reiff $A$, Silverman ED. Systemic lupus erythematosus in childhood. Rheum Dis Clin North Am 2002; 28: 561-77.

8. Mina R, Brunner HI. Pediatric Lupus - Are there differences in presentation, genetics, response to therapy, and damage accrual compared with adult lupus? Rheum Dis Clin N Am 2010; 36: 53-80.

9. Liphaus BL, Goldberg AC, Kiss MH, Silva CA. Analysis of human leukocyte antigens of class II-DR in Brazilian children and adolescents with systemic lupus erythematosus. Rev Hosp Clin Fac Med S. Paulo 2002; 57: 277-82.

10. Campos LM, Kiss MH, D'Amico EA, Silva CA. Antiphospholipid antibodies in 57 children and adolescents with systemic lupus erythematosus. Rev Hosp Clin Fac Med S. Paulo 2003; 58: 157-62.

11. Campos LM, Kiss MH, Scheinberg MA, Mangueira CL, Silva CA. Antinucleosome antibodies in patients with juvenile systemic lupus 
erythematosus. Lupus 2006; 15: 496-500.

12. Jesus AA, Silva CA, Carneiro-Sampaio $M$, Sheinberg $M$, Mangueira $C L$, Marie SK, Liphaus BL. Anti-C1q antibodies in juvenile-onset systemic lupus erythematosus. Ann N Y Acad Sci 2009; 1173: 235-8.

13. Miyamoto $M$, Ono E, Barbosa $C P$, Terreri MT, Hilário MO, Salomão R, Moraes-Pinto Ml. Vaccine antibodies and $\mathrm{T}$ - and B-cell interaction in juvenile systemic lupus erythematosus. Lupus 2011; 20: 736-44.

14. Amano MT, Ferriani VP, Florido MP, Reis ES, Delcolli MI, Azzolini AE, Assis-Pandochi Al, Sjöholm AG, Farah CS, Jensenius JC, Isaac L. Genetic analysis of complement $\mathrm{C} 1$ s deficiency associated with systemic lupus erythematosus highlights alternative splicing of normal $\mathrm{C} 1 \mathrm{~s}$ gene. Mol Immunol 2008; 45: 1693-702.

15. Liphaus BL, Kiss MH. The role of apoptosis proteins and complement components in the etiopathogenesis of systemic lupus erythematosus. Clinics (São Paulo) 2010; 65: 327-33.

16. Al-Mayouf SM, Al-Jumaah S, Bahabri S, Al-Eid W. Infections associated with juvenile systemic lupus erythematosus. Clin Exp Rheumatol 2001; 19: 748-50.

17. Canova EG, Rosa DC, Vallada MG, Silva CA. Invasive aspergillosis in juvenile systemic lupus erythematosus. A clinico-pathologic case. Clin Exp Rheumatol 2002; 20: 736.

18. Facó MM, Leone C, Campos LM, Febrônio MV, Marques HH, Silva CA. Factors associated to death in patients hospitalized with juvenile systemic lupus erythematosus. Braz J Med Biol Res 2007; 40: 993-1002.

19. Lee PP, Lee TL, Ho MH, Wong WH, Lau YL. Recurrent major infections in juvenile-onset systemic lupus erythematosus - a close link with longterm disease damage. Rheumatology (Oxford) 2007; 46: 1290-6.

20. França CM, Cavalcante EG, Ribeiro AS, Oliveira GT, Litvinov N, Silva CA. Disseminated histoplasmosis in a juvenile lupus erythematosus patient. Acta Reumatol Port 2012; 37: 276-9. 
21. Silva MF, Ribeiro AS, Fiorot FJ, Aikawa NE, Lotito AP, Campos LM, et al. Invasive aspergillosis: a severe infection in juvenile systemic lupus erythematosus patients. Lupus 2012; 21: 1011-6.

22. Costa-Reis $P$, Nativ $S$, Isgro J, Rodrigues T, Yildirim-Toruner C, Starr A, et al. Major infections in a cohort of 120 patients with juvenile-onset systemic lupus erythematosus. Clin Immunol 2013; 149: 442-9.

23. Marques VL, Gomes RC, Viola GR, Maia MM, Durigon GS, Aikawa NE, et al. Pulmonary cryptococcosis in childhood systemic lupus erythematosus and Sjögren syndrome overlap: a rare opportunistic infection. Lupus 2013; 22: 1409-12.

24. Cavalcante EG, Montoni JD, Oliveira GT, Campos LM, Paz JA, Silva CA. Fatal cryptococcal meningitis in a juvenile lupus erythematosus patient. Rev Bras Reumatol 2014; 54: 155-8.

25. Lube G, Aikawa NE, Tacla M, Leal MM, Lourenço B, Silva LE, et al. Condyloma acuminatum by human papilloma virus infection in childhoodsystemic lupus erythematosus patients. Acta Reumatol Port 2014; 39: 182-7.

26. Ciftçi E, Yalçinkaya F, Ince E, Ekim M, lleri M, Orgerin Z, Fitöz S, Güriz $H$, Aysev AD, Dogru U. Pulmonary involvement in childhood-onset systemic lupus erythematosus: a report of five cases. Rheumatology (Oxford) 2004; 43: 587-91.

27. Danza A, Ruiz Irastorza G. Infection risk in systemic lupus erythemamtosus patients: susceptibility factors and preventive strategies. Lupus 2013; 22:1286-94.

28. Hochberg MC. Updating the American College of Rheumatology revised criteria for the classification of systemic lupus erhytematosus. Arthrits Rheum 1997;40:1725.

29. Silva CA, Avcin T, Brunner HI. Taxonomy for systemic lupus erythematosus with onset before adulthood. Arthritis Care Res (Hoboken) 2012; 64: 1787-93.

30. De Pauw B, Walsh TJ, Donnelly JP, Stevens DA, Edwards JE, Calandra $\mathrm{T}$, et al. Revised definitions of invasive fungal disease from the European 
Organization for Research and Treatment of Cancer/Invasive Fungal Infections Cooperative Group and the National Institute of Allergy and Infectious Diseases Mycoses Study Group (EORTC/MSG) Consensus Group. Clin Infect Dis 2008; 46: 1813-21.

31. Hoenigl M, Strenger V, Buzina W, Valentin T, Koidl C, Wölfler A, et al. European Organization for the Research and Treatment of Cancer/Mycoses Study Group (EORTC/MSG) host factors and invasive fungal infections in patients with haematological malignancies. J Antimicrob Chemother 2012; 67: 2029-33.

32. American College of Rheumatology Ad Hoc committee on neuropsychiatric Lupus Syndromes. The American College of Rheumatology nomenclature and case definitions for neuropsychiatric lupus syndromes. Arthritis Rheum 1999; 42: 599-608.

33. Avcin T, Cimaz R, Rozman B. The Ped-APS Registry: the antiphospholipid syndrome in childhood. Lupus 2009; 18: 894-9.

34. National High Blood Pressure Education Program Working Group on High Blood Pressure in Children and Adolescents. The fourth report on the diagnosis, evaluation, and treatment of high blood pressure in children and adolescents. Pediatrics 2004; 114: 555-76.

35. Chan JC, Williams DM, Roth KS. Kidney failure in infants and children. Pediatr Rev 2002; 23: 47-60.

36. Akcan-Arikan A, Zappitelli M, Loftis LL, Washburn KK, Jefferson LS, Goldstein SL. Modified RIFLE criteria in critically ill children with acute kidney injury. Kidney International 2007; 71: 1028-35.

37. National Kidney Foundation. K/DOQI clinical practice guidelines for chronic kidney disease: evaluation, classification, and stratification. Am J Kidney Dis 2002; 39: S1-266.

38. Brandt JT, Triplett DA, Alving B, Scharrer I. Criteria for the diagnosis of lupus anticoagulants: an update. On behalf of the Subcommittee on Lupus Anticoagulant/Antiphospholipid Antibody of the Scientific and Standardisation Committee of the ISTH. Thromb Haemost 1995; 74: 1185-90. 
39. Gladman DD, Ibañez D, Urowitz MB. Systemic lupus erythematosus disease activity index 2000. J Rheumatol 2002; 29: 288-91.

40. Gladman D, Ginzler E, Goldsmith C, Fortin P, Liang M, Urowitz M, et al. The development and initial validation of the Systemic Lupus International Collaborating Clinics/American College of Rheumatology damage index for systemic lupus erythematosus. Arthritis Rheum 1996; 39: 363-9.

41. Barber CE, Barnabe C. Another consequence of severe lupus: invasive fungal disease. J Rheumatol 2012; 39: 1772-4.

42. Jesus AA, Jacob CM, Silva CA, Dorna M, Pastorino AC, CarneiroSampaio M. Common variable immunodeficiency associated with hepatosplenic T-cell lymphoma mimicking juvenile systemic lupus erythematosus. Clin Dev Immunol 2011; 2011: 428703.

43. Wang LR, Barber CE, Johnson AS, Barnabe C. Invasive fungal disease in systemic lupus erythematosus: A systematic review of disease characteristics, risk factors, and prognosis. Semin Arthritis Rheum 2014; 44: 325-30.

44. Chen HS, Tsai WP, Leu HS, Ho HH, Liou LB. Invasive fungal infection in systemic lupus erythematosus: an analysis of 15 cases and a literature review. Rheumatology (Oxford) 2007; 46: 539-44.

45. Kim HJ, Park YJ, Kim WU, Park SH, Cho CS. Invasive fungal infections in patients with systemic lupus erythematosus: experience from affiliated hospitals of Catholic University of Korea. Lupus 2009; 18: 661-6.

46. Fan YC, Li WG, Zheng MH, Gao W, Zhang YY, Song LJ. Invasive fungal infection in patients with systemic lupus erythematosus: experience from a single institute of Northern China. Gene 2012; 506: 184-7.

47. Martínez-Martínez MU, Herrera-Van Oostdam D, Román-Acosta S, Magaña-Aquino $M$, Baranda-Cándido $L$, Abud-Mendoza $C$. Invasive fungal infections in patients with systemic lupus erythematosus. J Rheumatol 2012; 39: 1814-8. 
48. Vinicki JP, Catalan Pellet S, Pappalardo C, Cruzat VC, Spinetto MA, et al. Invasive fungal infections in Argentine patients with systemic lupus erythematosus. Lupus 2013; 22: 892-8.

49. Colombo AL, Guimarães T, Silva LR, de Almeida Monfardini LP, Cunha AK, Rady $P$, et al. Prospective observational study of candidemia in São Paulo, Brazil: incidence rate, epidemiology, and predictors of mortality. Infect Control Hosp Epidemiol 2007; 28: 570-6.

50. Gonzalez-Crespo MR, Gomez-Reino JJ. Invasive aspergillosis in systemic lupus erythematosus. Semin Arthritis Rheum 1995; 24: 304-14.

51. Brunner HI, Gladman DD, Ibañez D, Urowitz MD, Silverman ED. Difference in disease features between childhood-onset and adult-onset systemic lupus erythematosus. Arthritis Rheum 2008; 58: 556-62.

52. Groll AH, Castagnola E, Cesaro S, Dalle JH, Engelhard D, Hope W, et al. Fourth European Conference on Infections in Leukaemia (ECIL-4): guidelines for diagnosis, prevention, and treatment of invasive fungal diseases in paediatric patients with cancer or allogeneic haemopoietic stem-cell transplantation. Lancet Oncol 2014; 15: $327-40$.

53. Yeh TC, Liu HC, Hou JY, Chen KH, Huang TH, Chang CY, et al. Severe infections in children with acute leukemia undergoing intensive chemotherapy can successfully be prevented by ciprofloxacin, voriconazole, or micafungin prophylaxis. Cancer 2014; 120: 1255-62. 


\section{Anexo I}

"A multicenter study of invasive fungal infections survey in 852 childhood-onset systemic lupus erythematosus patients"

Submetido para revista The Journal of Rheumatology. 